\title{
Quebra de dormência em sementes de Sesbania virgata (Cav.) Pers.
}

\author{
Treatments to break dormancy in Sesbania virgata (Cav.) Pers seeds \\ Paulo Eduardo de Menezes Silva ${ }^{1 *}$, Etenaldo Felipe Santiago ${ }^{2}$, Danilo de Menezes Daloso ${ }^{1}$, \\ Eder Marques da Silva ${ }^{3}$, Jeferson Oliveira Silva ${ }^{3}$
}

\section{RESUMO}

Sementes de espécies florestais que possuem tegumentos rígidos freqüentemente apresentam consideráveis problemas para a produção de mudas, por quanto que seus tegumentos duros e impermeáveis à água dificultam e retardam a germinação. Nesse sentido, esse trabalho objetivou determinar a metodologia mais eficiente para superação da dormência em sementes de Sesbania virgata (cav.) Pers., uma espécie com potencial para recomposição de áreas degradadas. Os tratamentos pré-germinativos utilizados foram imersão em ácido sulfúrico por 5, 10, 20 ou 30 minutos, escarificação mecânica ou choque térmico. Após a escarificação, as sementes de todos os tratamentos foram submetidas aos testes germinativos em câmaras de germinação a $25^{\circ} \mathrm{C}$ e fotoperíodo de $14 \mathrm{~h}$. $\mathrm{O}$ tratamento com escarificação mecânica demonstrou o maior percentual germinativo (98\%), seguida da escarificação química por 30 minutos (57\%). O tratamento térmico apesar de obter um maior percentual germinativo do que o controle apresentou desenvolvimento anormal de plântulas, não sendo, pois, recomendado para sementes de $S$. virgata. Diante dos parâmetros utilizados neste estudo, recomenda-se o uso da técnica de escarificação mecânica para a superação da dormência de sementes de $S$. virgata.

Palavras chave: Sesbania virgata, latencia, germinación de semillas.

\begin{abstract}
Forest species with hard seeds often pose considerable problems to nursery managers because their hard and impermeable seed coats hinder and delay germination. Therefore, this work aimed to determine the most efficient, practical and low cost methodology to overcome dormancy in Sesbania virgata (Cav.) Pers. Seed, a specie with potential for recovery of degraded areas. The seeds were submitted to chemical scarification by immersion in sulfuric acid during a period of 5, 10, 20 and 30 minutes, mechanical scarification and heat treatment. After the scarification the seeds were submitted to germ test in germination chambers at $25^{\circ} \mathrm{C}$ and photoperiod of $14 \mathrm{~h}$. Treatment with mechanical scarification showed the highest germination percentage (98\%), followed by chemical scarification for 30 minutes (57\%). The heat treatment in spite of obtaining a higher germination percentage than the controls had abnormal development of seedlings and is not therefore recommended for seeds of $\mathrm{S}$. virgata. From the parameters utilized in this study, we recommend the use of the mechanical scarification to overcome dormancy of $\mathrm{S}$. virgata seeds.
\end{abstract}

Key words: Sesbania virgata, dormancy, seed germination

\section{Introdução}

Sesbania virgata (Cav.) Pers. (sesbânia), é uma espécie pioneira, da família Leguminosae, ocorrente em campos alagáveis, solos arenosos ou argilosos (Braggio et al., 2002) e com potencial para a revegetação de áreas degradadas (Pott e Pott, 1994, Chaves et al., 2003), devido a disponibilidade de sementes (Samôr, 1999) e por apresentarem dormência tegumentar, a qual garante a sobrevivência da mesma até que encontre condições favoráveis para os processos de germinação e estabelecimento das plântulas (Roberts, 1981).

As sementes de espécies do gênero Sesbania apresentam diferentes níveis de dormência (Camargos et al., 2008). Nesse sentido, estudos acerca da quebra de dormência de sementes de $S$. virgata podem fornecer informações valiosas para as práticas de

1 Universidade Federal de Viçosa, Departamento de Biologia Vegetal, Programa de Pós-Graduação em Fisiologia Vegetal Viçosa-MG. E-mail: paulo.menezes@ufv.br, danilo_biologo@yahoo.com.br

2 Universidade Estadual de Mato Grosso do Sul, Centro Integrado de Análise e Monitoramento Ambiental, Laboratório de Ecologia, Dourados-MS. E-mail: felipe@uems.br, oliveria_jfs@hotmail.com

3 Universidade Estadual Paulista Júlio de Mesquita Filho, Instituto de Biociências, Departamento de Genética, Botucatu-SP. E-mail: dida24horas@hotmail.com

* Autor para correspondência 
manejo dessa espécie, fortalecendo sua utilização em projetos de reposição de cobertura vegetal em áreas degradadas.

Em sementes dormentes, a inviabilidade do embrião para retomar o crescimento após a embebição pode ser causada por uma série de fatores, como dormência fisiológica, que afeta direta ou indiretamente o metabolismo dos carboidratos, das proteínas e de outras reservas das sementes durante o processo germinativo (Vieira et al., 2000), e/ou dormência tegumentar, relacionada ao impedimento da entrada de água e/ou gases ao embrião (Hilhorst, 1995, Carvalho e Nakagawa, 2000, Baskin e Baskin, 2004). Veazey et al. (2000) relatam que o desenvolvimento de métodos para escarificação de sementes dormentes torna-se necessário para que seja manifestado o potencial real do lote de sementes tanto no campo como em testes para avaliação da qualidade fisiológica.

A dormência é um importante fator adaptativo e, portanto, um mecanismo de sobrevivência da espécie em determinadas condições, visto que retarda a germinação, distribuindo-a no tempo (Popinigis, 1977). No entanto, espécies que possuem sementes dormentes, comumente propiciam uma desuniforme produção de plântulas, dificultando a produção de mudas em viveiros e o planejamento dos plantios (Mattei, 1999). Dessa maneira, o conhecimento do mecanismo de dormência e da superação desta constitui fatores de elevada relevância na implantação de viveiros, visando a otimização da produção de mudas.

A quebra da dormência é regulada por interações complexas entre fatores ambientais e genéticos pouco conhecidos (Gluber et al., 2005). Dentro dessa perspectiva, os métodos de escarificação mecânica, química e física vêm sendo largamente utilizados como tratamentos pré-germinativos para otimizar o processo de germinação de sementes dormentes. Dentre esses, a escarificação mecânica tem sido eficientemente utilizada para quebrar a dormência de sementes de espécies da família Leguminosae, tais como Cassia grandis (Lobato, 1969), Erythrina speciosa (Carvalho et al., 1980), Peltophorum dubium (Bianchetti e Ramos, 1981b) e Enterolobium contortisiliquum (Cândido et al., 1982). Não obstante, a escarificação química também foi eficaz para superar a dormência de sementes dessa família, como Astragalus hamosus (Patane e Gresta, 2006), Schyzolobium excelsum (Freitas e Cândido, 1972),
Hymenaea courbaril e H. parvifolia (Carpanezzi e Marques, 1981), E. contortisiliquum (Cândido et al., 1982), Colubrina glandulosa (Queiroz, 1982) e P. dubium (Bianchetti e Ramos, 1981a, Guerra et al., 1982).

Além do tratamento químico, a escarificação física realizada com água a altas temperaturas tem sido aplicada visando à quebra da dormência de várias sementes de leguminosas. Esse método apresenta um baixo custo, sendo recomendado para sementes de Delonix regia (Murakami, 1976) e Schyzolobium parahybum (Bianchetti e Ramos, 1981a, Cândido et al., 1981).

Diante do supra exposto, esse trabalho objetivou estudar o comportamento germinativo de Sesbania virgata sob diferentes tratamentos de escarificação, a fim de aumentar o conhecimento sobre a biologia desta espécie.

\section{Materiais e Métodos}

Os experimentos foram conduzidos no Laboratório de Ecologia do Centro Integrado de Analise Ambiental-CInAM da Universidade Estadual de Mato Grosso do Sul, Dourados-MS.

Frutos de Sesbania virgata (Leguminosae) foram coletadas na Base de Estudos do PantanalBEP (19 34' 37' S e 57 00' 42" W), em Miranda - MS. As sementes foram separadas manualmente dos frutos e acondicionadas entre duas folhas de papel de filtro, à sombra, por um período de quinze dias. Os testes de germinação foram conduzidos em câmara de germinação tipo BOD, regulada a $25^{\circ} \mathrm{C}$, com fotoperíodo de 14 horas, por 30 dias.

Para superação de dormência das sementes, utilizaram-se técnicas de escarificação química, térmica e mecânica. Na escarificação química, as sementes foram embebidas em $20 \mathrm{~mL}$ de ácido sulfúrico (98\%) sob diferentes tempos de imersão (5, 10, 20 ou 30 minutos). Decorridos os tempos estabelecidos para cada tratamento, a solução ácida foi drenada e as sementes lavadas superficialmente em água corrente durante 5 minutos.

Nos testes com escarificação mecânica, as sementes foram friccionadas manualmente em uma lixa, raspando a parte superior e oposta ao eixo do embrião (Brasil, 1992), como forma de evitar danos ao embrião. A escarificação térmica foi realizada submetendo as sementes a um choque térmico, imergindo em água fervente $\left(100^{\circ} \mathrm{C}\right)$ por 10 minutos e logo após em água gelada. 
Posteriormente à aplicação dos tratamentos, as sementes foram secas superficialmente sobre papel filtro, sendo utilizadas, em seguida, nos testes de germinação.

Neste estudo, foi empregado o delineamento inteiramente casualizado, com seis tratamentos e quatro repetições, sendo cada parcela experimental constituída de 25 sementes. Calcularam-se a porcentagem e a freqüência relativa de acordo com as fórmulas descritas em Labouriau (1983) e o índice de velocidade de germinação (IVG) segundo Maguire (1962). Os dados foram submetidos à análise de variância e as médias foram comparadas pelo teste de Tukey (5\% de probabilidade) (Gomes, 1987).

\section{Resultados e Discussão}

Na ausência de tratamentos pré-germinativos (lote controle), não foram observadas sementes germinadas durante o período experimental (Figura 1), fato este que comprova a presença de dormência em sementes de Sesbania virgata.

Dentre os tratamentos químicos utilizados nesse estudo, a maior porcentagem de germinação foi obtida através da imersão das sementes em ácido sulfúrico por 30 minutos, sendo que a imersão por 20 minutos também obteve bons resultados (Figura 1). Embora a imersão por 5 e 10 minutos tenham apresentado menores valores de germinação quando comparado aos outros tratamentos químicos, estes já se mostraram eficientes para provocar a germinação das sementes.

Considerando os resultados obtidos com a imersão das sementes em ácido sulfúrico, pode-se

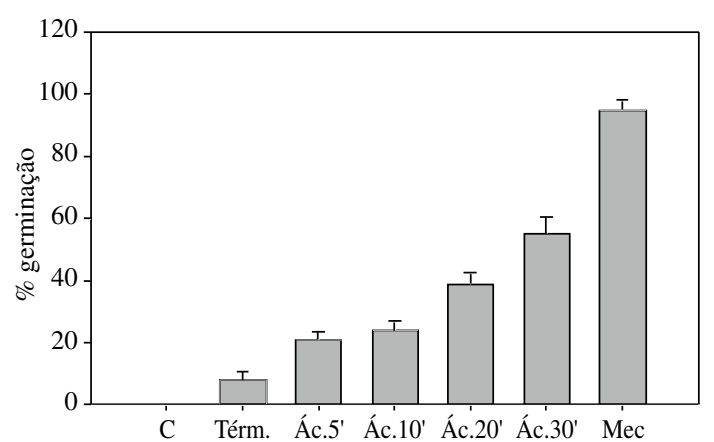

Figura 1. Porcentagem média de germinação de sementes de Sesbania virgata do lote controle (C) ou submetidas à escarificação térmica (Térm.), escarificação mecânica (Mec), escarificação com ácido sulfúrico (98\%) por 5 (Ác.5'), 10 (Ác.10'), 20 (Ác.20’) ou 30 (Ác.30') minutos. afirmar que este foi um teste eficaz na superação da dormência, porém com o aumento do tempo de imersão, talvez seja possível atingir melhores resultados, visto que o tratamento com imersão por 30 minutos não apresentou plântulas defeituosas. Esses resultados, juntamente com os obtidos por Bakke e Gonçalves (1984) e Bianchetti (1981), comprovam a eficiência de tratamentos com ácido sulfúrico para superar a dormência de sementes de algumas espécies. No entanto, esse tratamento não deve ser utilizado indiscriminadamente para sementes de quaisquer espécies, pois esse tratamento pode danificar seriamente as sementes testadas (Maeda e Lago, 1986).

A escarificação mecânica superou todos os outros tratamentos utilizados neste estudo, para todas as variáveis analisadas, obtendo um percentual de germinação de $95 \%$, muito superior ao melhor tratamento obtido com a escarificação química (57\%) (Figura 1).

O método de escarificação mecânica também foi o mais eficiente em otimizar a porcentagem de germinação em batata-de-purga (Operculina macrocarpa) (Filho et al., 2002) e jatobá-curuba (Hymenaea intermedia) (Cruz et al., 2001). A escarificação mecânica pode ser efetiva para promover a germinação da semente por facilitar a entrada de água para o eixo embrionário, permitindo, assim, o início do processo germinativo com a hidratação do embrião (Áquila e Fett Neto, 1988).

Os resultados obtidos nesse estudo demonstraram a eficiência da escarificação através da utilização de materiais abrasivos para a quebra de dormência de sementes, fato também demonstrado em sementes de Erytrina velutina Willd. (Silva e Matos, 1993-1994), Leucaena diversifolia (Schltoll.) Benth. (Bertalot e Nakagawa, 1998), Passiflora alata Dryand (Rossetto et al., 2000), Bauhinia monandra e B. ungulata (Alves et al., 2000).

Entre os métodos utilizados para superação da dormência tegumentar, a escarificação mecânica é uma técnica freqüentemente utilizada e constitui a opção mais prática e segura para pequenos agricultores (Hermansen et al., 2000), por ser um método simples, de baixo custo e eficaz para promover uma rápida e uniforme germinação. No entanto, deve ser efetuada com cuidado para evitar que a escarificação excessiva possa causar danos ao tegumento e diminuir as taxas de germinação (McDonald e Copeland, 1997). 


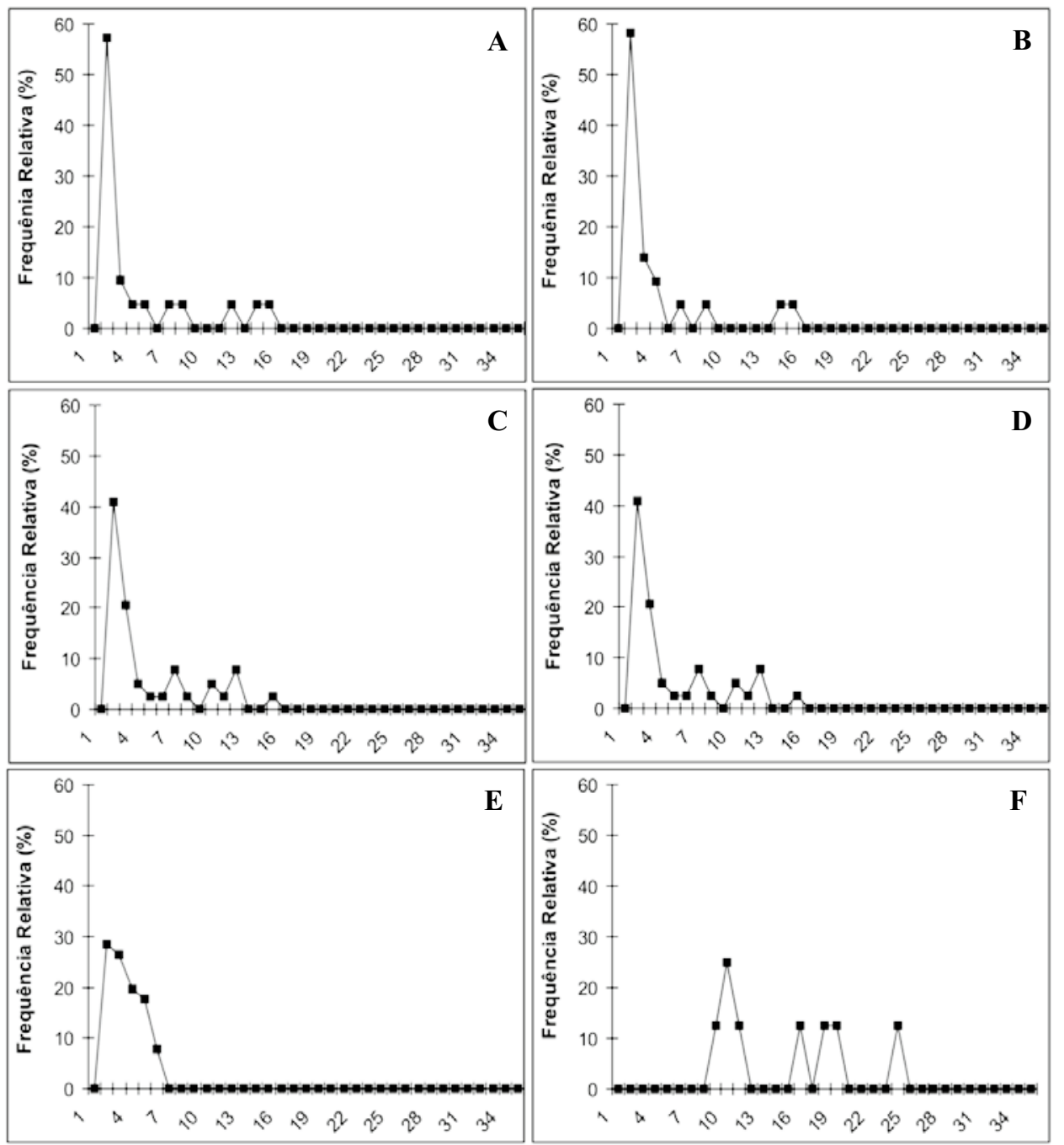

Figura 2. Freqüência relativa de germinação para sementes de Sesbania virgata submetidas a diferentes tratamentos de escarificação. A, B, C e D-Escarificação Química por 5', 10', 20’ ou 30', respectivamente, E-Escarificação Mecânica, F-Escarificação Térmica.

A escarificação térmica foi a menos eficaz dentre os tratamentos analisados, obtendo um percentual germinativo de $8 \%$, valor este muito abaixo dos obtidos com os outros métodos escarificantes testados nesse estudo. Em adição, sob esse tratamento, todas as sementes germinadas geraram plântulas deformadas. Resultados semelhantes foram obtidos por Varela et al., (1991) e Alves et al., (2004), em que a imersão em água a temperatura de $50^{\circ}$ e $80^{\circ} \mathrm{C}$, respectivamente, geraram baixo percentual de germinação em sementes de Stryphnodendron pulcherimum (Willd.) Hochr e Bauhinia divaricata $\mathrm{L}$.
Azania et al. (2003) utilizando a imersão de sementes em água quente $\left(50{ }^{\circ} \mathrm{C}\right)$ por 40 minutos, relataram um aumento na porcentagem de germinação em sementes de I. grandifolia, M. cissoides e $M$. aegyptia. Nesse contexto, a utilização de temperaturas menores que a utilizada nesse estudo $\left(100^{\circ} \mathrm{C}\right)$, possivelmente contribuiriam para aumentar o percentual de germinação das sementes de $S$. virgata, uma vez que altas temperaturas podem danificar as sementes, fato que provavelmente tenha ocorrido às sementes de $S$. virgata sob imersão em água a $100^{\circ} \mathrm{C}$.

As sementes submetidas à escarificação mecânica apresentaram o maior índice de velocidade de 
Tabela 1. Valores médios para porcentagem $(\mathrm{G} \%)$ e índice de velocidade de germinação (IVG) de sementes de Sesbania virgata submetidas a diferentes tratamentos.

\begin{tabular}{lcc}
\hline Tratamento & G $(\%)$ & IVG $\left(\right.$ dias $\left.^{-1}\right)$ \\
\hline Controle & Of & 0f \\
Ac. 5' & $21 \mathrm{~d}$ & $0,232 \mathrm{c}$ \\
Ac. $10{ }^{\prime}$ & $24 \mathrm{~d}$ & $0,251 \mathrm{~b}$ \\
Ac. $20^{\prime}$ & $39 \mathrm{c}$ & $0,209 \mathrm{~d}$ \\
Ac. $30^{\prime}$ & $57 \mathrm{~b}$ & $0,207 \mathrm{~d}$ \\
Esc. Mecânica & $95 \mathrm{a}$ & $0,285 \mathrm{a}$ \\
Esc. Térmica & $8 \mathrm{e}$ & $0,068 \mathrm{e}$ \\
\hline
\end{tabular}

Diferentes letras, em coluna, demonstram diferença estatística pelo teste Tukey $(\mathrm{p}<5 \%)$.

germinação (IVG) (Tabela 1), demonstrando que esse método, por provocar fissuras no tegumento das sementes, permite a embebição dessas e a aceleração do início do processo de germinação (Frank e Baseggio, 1998). Assim, esse tratamento, além de ser prático e eficiente, proporcionou maior área de contato entre a semente e o substrato úmido, o que ocasionou eficiente absorção de água e assegurou os melhores resultados de germinação e IVG.

Nos tratamentos com ácido sulfúrico (98\%), os maiores IVG foram observados em 5 e 10 min de imersão (Tabela 1), embora esses tenham apresentado menores porcentagens de germinação entre os tratamentos químicos (Figura 1). A escarificação térmica apresentou os menores IVG (Tabela 1), isso pode ser explicado pelos possíveis danos fisiológicos que as altas temperaturas provavelmente causaram aos embriões das sementes.

O caráter unimodal do gráfico de freqüência relativa de germinação sob escarificação mecânica (Figura $2 \mathrm{E}$ ) demonstrou uma grande uniformidade de germinação após esse tratamento, em que a germinação ocorreu de maneira rápida e uniforme. Já após os tratamentos químicos, os gráficos de freqüência relativa de germinação tornam-se polimodais (Figuras $2 \mathrm{~A}, \mathrm{~B}, \mathrm{C}$ e D), em que se observam picos germinativos entre o $2^{\circ}$ e $3^{\circ}$ dia, no entanto, após este período, as sementes continuaram germinando de maneira desuniforme, demonstrando maior heterogeneidade no tempo de germinação. O tratamento térmico apresentou pico de germinação no $12^{\circ}$ dia (Figura $2 \mathrm{~F}$ ), ao passo que na escarificação mecânica o pico ocorreu no $2^{\circ}$ dia (Figura 2 E).

Em condições naturais, é esperado o caráter adaptativo da heterogeneidade na germinação de sementes conferido, principalmente, pela dormência. A heterogeneidade da germinação em relação ao tempo, tende a distribuir a germinação ao longo do tempo, aumentando dessa forma a possibilidade de que pelo menos algumas sementes germinem sob condições ótimas. Dentro dessa perspectiva, a quebra da dormência e a distribuição da germinação ao longo do tempo possuem grandes efeitos sobre a sobrevivência e o estabelecimento de plântulas (Silvertown, 1987), dessa forma, a distribuição da germinação constitui um comportamento adaptativo, porém de caráter pouco específico, pois a quebra da dormência não determina a época provável de germinação.

Embora os tratamentos químicos tenham sido eficientes para quebrar a dormência das sementes de $S$. virgata, a distribuição da germinação após esses tratamentos mostrou-se muito desuniforme. Assim, baseado na uniformidade, no IVG a na porcentagem de germinação de sementes de $S$. virgata submetidas à escarificação mecânica, conclui-se que esse método foi o método mais eficaz para otimizar a germinação de sementes e a produção de mudas dessa espécie.

\section{Conclusões}

Através do estudo do comportamento germinativo de Sesbania virgata (cav.) Pers. Após diferentes tratamentos pré-germinativos, verificou-se que as sementes dessa espécie apresentam dormência, o que as tornam sementes impermeáveis a água, sugerindo uma barreira à germinação. Dentre as condições experimentais utilizadas, o melhor tratamento de quebra de dormência foi a escarificação mecânica, seguido da escarificação química com ácido sulfúrico (98\%), sendo os métodos mais eficientes para superar a dormência de sementes de S. virgata. 


\section{Referências Citadas}

Alves, M.C.S.; Medeiros-Filho, S.; Andrade-Neto, M.; Teófilo, E.M.

2000 Superação da dormência em sementes de Bauhinia monandra Britt e Bauhinia ungulata L. - Caesalpinoideae. Revista Brasileira de Sementes, Londrina, v. 22, n. 2, pp. 139-144.

Alves, A.U.; Dornelas, C.S.M.

2004 Superação da dormência em sementes de Bauhinia divaricata L. Acta Botânica Brasílica, São Paulo, v. 18, n. 4, pp. 871-879.

Áquila, M.S.A.; Fett Neto, A.G.

1988 Influência de processos de escarificação na germinação e crescimento inicial de Leucaena leucocephala (Lam.) DeWit. Revista Brasileira de Sementes, Brasília, v. 10, n. 1, pp. 73-85.

Azania, A.A.P.M.; Marques, M.O.; Pavani, M.C.M.D. \& Azania, C.A.M.

2003 Germinação de sementes de Sida rhombifolia e Brachiaria decumbens influenciada por vinhaça, flegmaça e óleo de fusel. Planta Daninha, Viçosa-MG, v. 21, n. 3, pp. 443449.

Bakke, O.A.; Golçalves, W.

1984 Quebra de dormência de sementes de algaroba (Prosopis juliflora DC). In: Simpósio florestal internacional, 1984. Anais... pp. 65-82.

Baskin, J.M. and Baskin, C.C.

2004 A classification system for seed dormancy. Seed Science Research. 14: 1-16.

Bertalot, M.J.A.; Nakagawa, J.

1998 Superação da dormência em sementes de Leucaena diversifolia (Schlecht.). Bentham K156. Revista Brasileira de Sementes, v. 20, n. 1, pp. 39-42.

Bianchetti, A.

1981 Comparação de tratamentos para superar a dormência de sementes de bracatinga (Mimosa scabrella Bentham). Boletim de Pesquisa Florestal, Curitiba, (a): 57-68.

Bianchetti, A. \& Ramos, A.

1981a Quebra de dormência de sementes guapuruvu (Schizolobium parahyba) (Vellozo) Blake. Boletim de Pesquisa Florestal, Curitiba, 3: 69-76.

Bianchetti, A. \& Ramos, A

1981b Quebra de dormência de sementes de canafístula Peltophorum dubium (Spreng.) Taubert. Resultados preliminares. Boletim de Pesquisa Florestal, Curitiba, 3: 87-95.

Braggio, M.M., Lima, M.E.L., Veasey, E.A., Haraguchi, M. 2002 Atividades farmacológicas das folhas da Sesbania virgata (CAV.) PERS. Arquivos do Instituto. Biológico, São Paulo, v. 69 , n. 4 , pp. $49-53$, out./dez.

BRASIL,

1992 Ministério da Agricultura e Reforma Agrária. Regras para análise de sementes. Brasília: SNDA/DNDV/CLAV, $365 \mathrm{p}$.

Camargos, V.N., Carvalho, M.L.M., Araujo, D.V., Magalhaes, FH.L.

2008 Superação da dormência e avaliação da qualidade fisiológica de sementes de Sesbania virgata. Ciência e agrotecnologia, vol. 32, n. 6, pp. 1858-1865.

Cândido, J.F.; Condë, A.R.; Silva, R.F.; Maria, J. \& Lëdo, A.A.M.
1981 Estudo da causa da dormência em sementes de guapuruvu (Schizolobium parahybum (Vell.) e métodos para sua quebra. Revista Árvore, Viçosa, 5(2): 224-32.

Cândido, J.F.; Silva, R.F.; Condë, A.R. \& Lêdo, A.A.M

1982 Orelha-de-negro (Enterolobium contortisiliquum (Vell.) Morong: dormência e métodos para a sua quebra. Revista Árvore, Viçosa, 6(2): 104-10.

Carpanezzi, A.A.; Marques, L.C.T.

1981 Germinação de sementes de jutaiacú (Hymenaea courbaril L.) e de jutaí-mirim (Hymenaea parvifolia Huber) escarificadas com ácido sulfúrico comercial. Belém: EMBRAPA-CPATU, Circular Técnica,19. 15 p.

Carvalho, N.M.; Demattê, M.E.S.P. \& Graziano, T.T.

1980 Germinação de sementes de essências florestais nativas. I. suinã ou mulungu (Erythryna speciosa Andr.), Revista Brasileira de Sementes. Brasília, 2(1): 81-7.

Carvalho, N.M. \& Nakagawa, J.

2000 Sementes: ciência, tecnologia e produção. $4^{\mathrm{a}}$ ed. Funep, Jaboticabal.

Chaves, L. DE L.B.; Carneiro, J.G. DE A.; Barroso, D.G.E.; Leles, P.S.; Dos S.

2003 Efeitos da inoculação com rizóbio e da adubação nitrogenada na produção de mudas de Sesbania em substrato constituído de resíduos agroindustriais. Revista Árvore. Viçosa, v. 27. n. 4, pp. 443-449.

Cruz, E.D.; Martins, F.O.; Carvalho, J.E.U.

2001 Biometria de frutos de jatobá-curuba (Hymenaea intermedia Ducke, Leguminosae-Caesalpinoideae). Revista Brasileira de Botânica 24(2): 161-165.

Filho, S. M.; França, E.A. DE; Innecco, R.

2002 Germinação de sementes de Operculina macrocarpa (L.) Farwel e Operculina alata (Ham.) Urban1. Revista Brasileira de Sementes, vol. 24, n. 2, pp. 102-107.

Franke, L.B; Baseggio, J.

1988 Superação da dormência em esmentes de Desmodium incamum DC. e Lathyrus nervosus Lam. Revista Brasileira de Sementes, v. 20, n. 2, pp. 420-424.

Freitas, J.A.C. \& Cândido, J.F

1972 Tratamento químico para abreviar germinação de sementes de guapuruvu (Schyzolobium excelsum Vog.) e de mamoneira (Tachigalia multijuga Bth). Seiva, Viçosa, (32): 1-10.

Gomes, F.P.

1987 Curso de estatística experimental. Piracicaba: ESALQ, $467 \mathrm{p}$.

Guerra, M.P.; Nodari, R.O.; Reis, A. \& Grando, J.L.

1982 Comportamento da canafístula (Peltophorum dubium (Sprengel) Taubert. em viveiro, submetida a diferentes métodos de quebra de dormência e semeadura. Boletim de Pesquisa Florestal., Curitiba, 5: 1-15.

Hilhorst, H. W. M.

1995 A critical update on seed dormancy. I. Primary dormancy. Seed Science Research, v. 5, n. 1, pp. 61-73.

Hermansen L.A.; Duryea M.L.; White, T.L. 2000 Viability in seed coat dormancy in Dimorphandra mollis. Seed Science. Technology, 28: 567-580.

Labouriau, L.G.

1983 A germinação das sementes. Secretaria Geral da Organização dos Estados Americanos, Washington. 
Lobato, R.C.

1969 Experimento sobre a germinação de Cassia grandis L.f. (Leguminosae-Caesalpinoideae) com aplicação de pré-tratamentos. Revista de Farmácia e Bioquímica da Amazônia, Belém, 16: 21.

Maeda, J.A \& Lago, A.A.

1986 Germinação de sementes de mucuna-preta após tratamento para superação da impermeabilidade do tegumento. Revista Brasileira de Sementes, 8 (1): 79-86.

Maguire, J.D.

1962 Speed of germination aid in selection and evaluation for seedling emergence and vigor. Crop Science, Madison, v. 2, n. 2, pp. 176-77.

Mattei, V.L.

1999 Efeito de tratamento em sementes dormentes de Acácia trinervis (Acácia longifólia Willd), sobre a germinação em laboratório, emergência e desenvolvimento inicial em viveiro. Revista Brasileira de Agrociência, v. 5, n. 3, pp. 185-189.

Murakami, M.T.

1976 Estudos de quebra de dormência de sementes de Delonix regia, Rafin (Flamboyant). Jaboticabal, FCAV/UNESP, 40 p. (Trabalho de Graduaçao).

McDonald, M.B.

1997 Copeland, L.O. Seed production: principles and practices. New Jersey: Chapmam \& Hall, 749 p.

Patane, C. and Gresta, F.

2006 Germination of Astragalus hamosus and Medicago orbicularis as affected by seed coat dormancy breaking techniques. J. Arid Environment, 67: 165-173.

Popinigis, F.

1977 Fisiologia da semente. Brasília: AGIPLAN, 189 p.

Pott, A., Pott, V. 1994 Plantas do pantanal. Brasília: EMBRAPA-SPL, 320 p.

Queiroz, M.H.

1982 Triagem densimétrica e quebra de dormência em Colubrina glandulosa Perkins var. Reitzu (M.C. Johnston) M.C. Johnston Silvicultura São Paulo, São Paulo, 16A (1): 307-11.
Roberts, H.A.

1981 Seed Banks in Soils.Advances in Applied Biology 6 (1): 1-55.

Rossetto, C.A.V.; Coneglian, R.C.C.; Nakagawa, J.; Shimizu, M.K.; Marin, V. A.

2000 Germinação de sementes de maracujá-doce (Passiflora alata Dryand) em função de tratamento pré-germinativo. Revista Brasileira de Sementes, Londrina, v. 22, n. 1, pp. 247-252.

Samôr, O.J.M.

1999 Comportamento de mudas de Sesbania virgata e Anadenanthera macrocarpa, produzidas em recipientes e substratos, destinadas à recuperação de áreas degradadas pela extração de argila. Campos de Goytacazes. Dissertação (Mestrado em Produção Vegetal), Universidade Estadual do Norte Fluminense.

Silva, L.M.M.; Matos, V.P.

1993-1994 Estudo sobre dormência de sementes de mulungu (Erythrina velutina Willd.): viabilidade e presença de inibidores. Ciência Agrícola, v. 2, n. 1, pp. 29-40.

Silvertown, J.W.

1987 Introduction to plant population ecology. N.Y. Longman Scientific \& Technical. 229 p.

Varela, V.P.; Brocki, E. \& Sá, S.T.V.

1991 Tratamentos prégerminativos de espécies da Amazônica IV. Faveira camuzê-Stryphnodendron pulcherimum (Willd.) Hochr-Leguminosae. Revista Brasileira de Sementes, Brasília. v. 13 , n. 2, pp. 87-89.

Veazey, E.A.; Freitas, J.C.T.; Schammass, E.A.

2000 Variabilidade da dormência de sementes entre e dentro de espécies de Sesbânia. Scientia Agricola, Piracicaba, v. 57, pp. 229-304.

Vieira, A.R.; Vieira, M. das G.G.C.; Oliveira, J.A. et al.

2000 Alterações fisiológicas e enzimáticas em sementes dormentes de arroz armazenadas em diferentes ambientes. Revista Brasileira de Sementes, v. 22, n. 2, pp. 53-61. 